\title{
Synthesis of Carbonated Hydroxyapatite Nanorods in Liquid Crystals
}

\author{
Daniella Dias Palombino de Campos, Celso Aparecido Bertran*
}

\author{
Chemistry Institute, University of Campinas, P.O. Box 6154, Campinas - SP, Brazil
}

Received: August 18, 2008; Revised: August 18, 2009

\begin{abstract}
Syntheses of calcium phosphate nanoparticles, carried out in systems formed from surfactant, oil and water, have resulted in materials with promising possibilities for application. The calcium phosphate particles were synthesized using two different liquid crystals, formed from Renex ${ }^{\mathrm{TM}}$, cyclohexane and a salts solution. The morphology of the nanoparticles synthesized in the liquid crystals is similar to that of hydroxyapatite particles that form bone mineral, where collagen fibers connect these particles so as to form a composite. Therefore, the synthesis of calcium phosphate nanoparticles in the systems used in this work can advance current understanding of mineralization processes that result in the formation of bone mineral.
\end{abstract}

Keywords: hydroxyapatite, synthesis, liquid crystals

\section{Introduction}

Hard materials that form the skeleton of vertebrates or the exoskeleton of invertebrates are quite often composites formed from an inorganic phase and an organic phase. For instance, the shells of mollusks are basically formed from proteins, polysacchacarides and calcium carbonate, and bones are mainly composed of calcium phosphates and collagen ${ }^{1,2}$.

The hard materials that compose skeletons are formed through a process known as "biomineralization", in which the organic phase causes the inorganic phase to crystallize. The organic phase controls the processes of nucleation and the crystal growth of the inorganic phase ${ }^{3}$.

In the specific case of bone formation, the hard material, called the bone matrix, is constituted of hydroxyapatite nanoparticles (HAP) produced in the organic phase, which is composed mainly of collagen. The hard tissue of bone is therefore a composite in which collagen organized into fibers connects HAP nanoparticles ${ }^{4,5}$

One of the models for the creation of composites that form the hard material of bone assumes that the organic phase self-organizes by segregating the precursor of the inorganic phase into "matrix vesicles" or "nanoreactors". Such nanoreactors control nucleation and growth processes of the inorganic phase, as well as the dimensions and morphologies of its particles ${ }^{3,6}$.

That model, for instance, could explain the formation of bone matrix, composed of rounded HAP particles of about $20 \mathrm{~nm}$ diameter that coat a structure formed from collagen fibers, or of the "clump" of seashells, formed from alternate layers of calcium carbonate particles and organic matrix in a brick-and-mortar kind of arrangement ${ }^{1,6}$.

Systems composed of surfactant-water-oil form self-aggregated structures such as liquid crystals, which have been employed as a medium that can mimic the nanoreactors from the mineralization model by performing the role of the organic phase, i.e. by controlling the nucleation and growth of inorganic phase particles ${ }^{7}$.

Therefore, the synthesis of calcium phosphate particles such as HAP within these self-aggregated structures can mimic the control exercised by the organic phase in the processes of nucleation and particle growth, which result in the formation of bone matrix ${ }^{7}$.

In this work, two liquid crystals composed of Renex, cyclohexane and a salts solution were employed in the production of nanorods formed from HAP. The morphologies of these nanorods suggest that they result from the agglomeration of rounded HAP particles, in a process similar to bone matrix formation, where HAP particle agglomerates coat collagen fibers.

\section{Materials and Methods}

\subsection{Preparation of liquid crystals}

For the preparation of liquid crystals, two different nonionic surfactants, both polyoxyethylene nonylphenylethers $\left[\mathrm{C}_{9} \mathrm{H}_{19} \mathrm{C}_{6} \mathrm{H}_{4}\left(\mathrm{OCH}_{2} \mathrm{CH}_{2}\right)_{\mathrm{n}} \mathrm{OH}\right]$ were used. Both are named Renex ${ }^{\mathrm{TM}}$ from Oxiteno/Brazil: in one $\mathrm{n}=6\left(\right.$ Renex $\left.-60^{\mathrm{TM}}\right)$ and in the other $\mathrm{n}=10\left(\right.$ Renex $\left.-100^{\mathrm{TM}}\right)$.

The 2 separate phases were prepared by mixing the reagents at room temperature, using the following compositions (in percentage of mass): $45.5 \%$ Renex $^{\mathrm{TM}}, 9 \%$ cyclohexane (Synth) and $45.5 \%$ salts solution. This composition is analogous to the diagram obtained for Triton X-100, a surfactant similar to Renex-100"TM,8. The term "salts solution" refers to the solution used as a source of $\mathrm{Ca}^{2+}$ and $\mathrm{PO}_{4}^{3-}$ for the synthesis of HAP, containing 1 mol.L $\mathrm{L}^{-1}$ calcium nitrate (Synth) and 0.6 mol.L $\mathrm{L}^{-1}$ phosphoric acid (Dinâmica), at $\mathrm{pH}$ 2.0. The phases containing Renex-60 and Renex-100 as surfactants were labeled LCR60 and LCR100, respectively.

\subsection{Synthesis of hydroxyapatite}

Following the mixing of the reagents, the liquid crystals were exposed to an ammonia (Synth) atmosphere in a closed system for 10 days at room temperature. $\mathrm{As} \mathrm{NH}_{3}$ permeates into the liquid crystals, the $\mathrm{pH}$ rises, causing HAP to precipitate within liquid crystaline phases. After this 10-day period, samples were heated in an oven at 


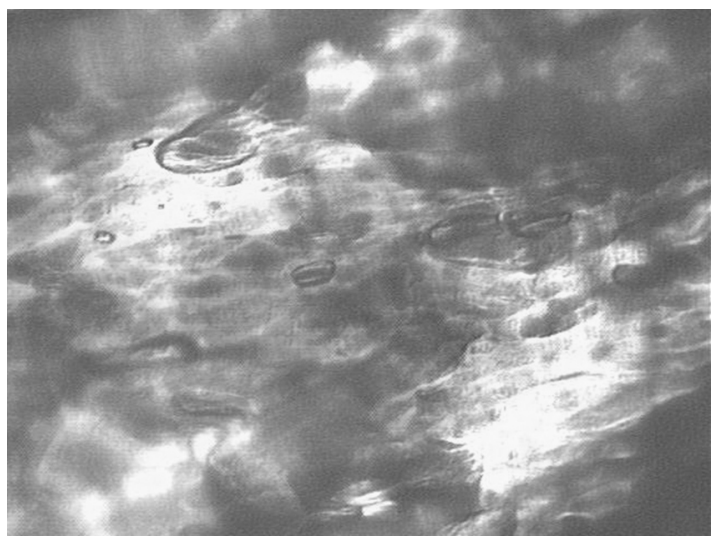

Figure 1. Birefringence observed in liquid crystals formed from Renex- $100^{\mathrm{TM}}$.

$100{ }^{\circ} \mathrm{C}$ for 3 hours, at a heating rate of $05^{\circ} \mathrm{C} / \mathrm{min}$, in order to eliminate excess $\mathrm{NH}_{3}$, water and cyclohexane, as well as to decompose the ammonium nitrate formed during neutralization by $\mathrm{NH}_{3}$. The first thermal treatment was slow in order to preserve the morphology of nanoparticles, which otherwise avoid change due to the rapid elimination of the volatile reagents. After treatment at $100{ }^{\circ} \mathrm{C}$, the oven temperature was increased over three hours to $500^{\circ} \mathrm{C}$. Samples were kept at this temperature for 3 days to remove the Renex.

\subsection{Characterization of the liquid crystals}

Both liquid-crystalline phases (formed from Renex-60 and -100) were identified by Deuterium Nuclear Magnetic Ressonance, i.e. ${ }^{2} \mathrm{H}-\mathrm{NMR}$ (VARIAN, Inova 500). For this purpose, samples were prepared as described above but $5 \%$ deuterium oxide $\left(\mathrm{D}_{2} \mathrm{O}\right.$ Cambridge Isotope ${ }^{\circledR}$ Laboratories, Inc.) was substituted for $5 \%$ of the salts solution.

The liquid crystals were also characterized by optical microscopy with plane-polarized light, i.e POM (NIKON ${ }^{\circledR}$, Eclipse E800), where the birefringence typical of such phases was detected.

\subsection{Characterization of hydroxyapatite}

The HAP nanoparticles obtained after thermal treatment at $500{ }^{\circ} \mathrm{C}$ were characterized by XRD, FTIR and SEM. The $\mathrm{Ca} / \mathrm{P}$ ratio of the calcium phosphate in the samples was determined by ICP-OES.

$\mathrm{X}$-ray diffraction (XRD) patterns of the particles were recorded on a Shimadzu XRD7000 diffractometer, operated at $40 \mathrm{kV}$ and $30 \mathrm{~mA}$, using $\mathrm{CuK}$ radiation $\alpha(\lambda=0.15418 \mathrm{~nm})$ over the range $05^{\circ}<2 \Theta<65^{\circ}$ at a $2 \%$ min acquisition speed.

Fourier Transform Infrared (FTIR) spectroscopy was performed on a Bomem B 100 Spectrometer with a resolution of $4 \mathrm{~cm}^{-1}$, using the typical $\mathrm{KBr}$ pellet technique.

Micrographs of the nanoparticles were obtained using a JSM6360-LV Scanning Electron Microscope. The electron beam was used with an accelerating voltage of $20 \mathrm{kV}$. Samples were mounted on a copper sample holder with carbon ribbon and coated with carbon and gold using a Bal-Tec ${ }^{\circledR}$ MCS 010 sputtering device.

$\mathrm{Ca} / \mathrm{P}$ ratios of the particles were obtained, after dissolution in nitric acid (Synth), using an Inductive Coupled Plasma - Optical Emission Spectrophotometer (Optima 3000DV).

\section{Results and Discussion}

\subsection{Identification of liquid crystals}

Liquid crystals prepared with Renex-60 and Renex-100 (LCR60 and LCR100) presented birefringence when observed by optical microscopy with plane-polarized light, before permeation with ammonia (Figure 1). The fact that birefringence was observed in both preparations shows that, in spite of the difference in the number of EO groups between the 2 surfactants utilized, the preparation of these systems following the composition obtained from the phase diagram of Triton X-100 ${ }^{8}$, resulted in liquid crystals in both cases.

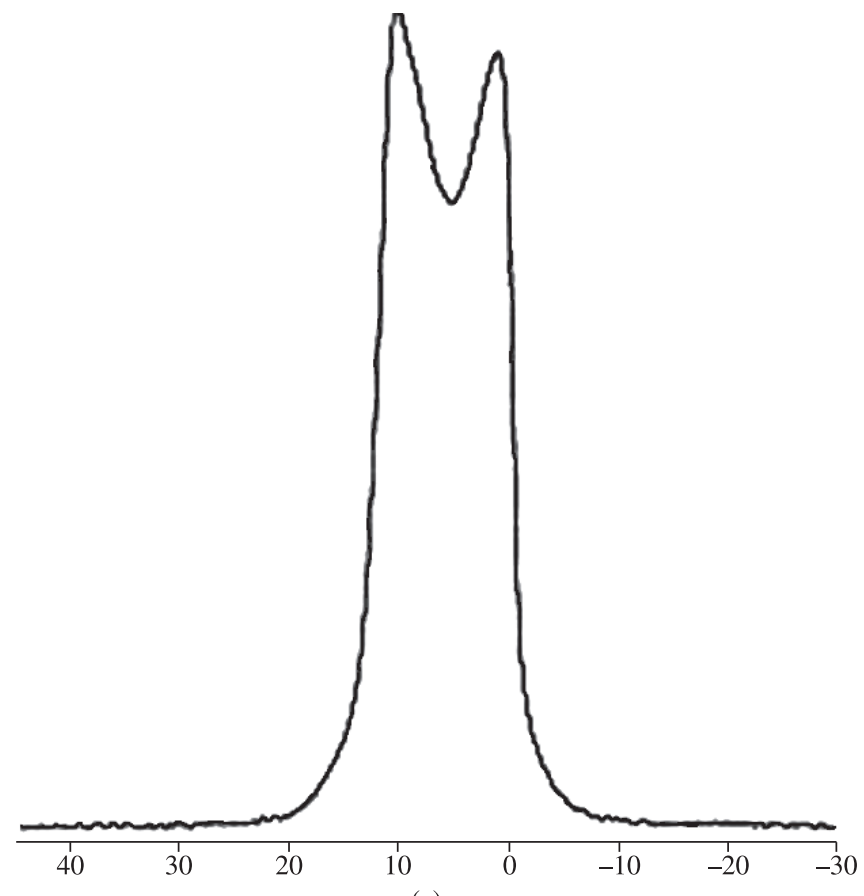

(a)

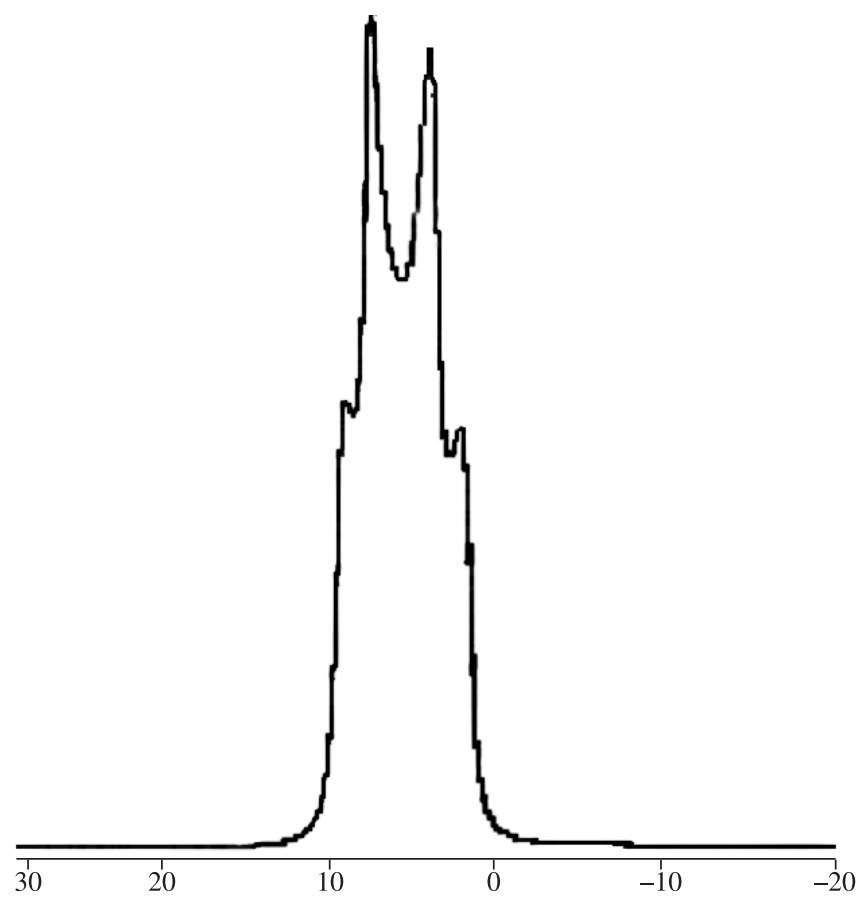

(b)

Figure 2. $2 \mathrm{H}$-NMR spectra for liquid crystal phases with a) Renex-60 ${ }^{\mathrm{TM}}$ and b) Renex-100 ${ }^{\mathrm{TM}}$. Both phases were enriched with $5 \% \mathrm{D}_{2} \mathrm{O}(\mathrm{w} / \mathrm{w})$. 


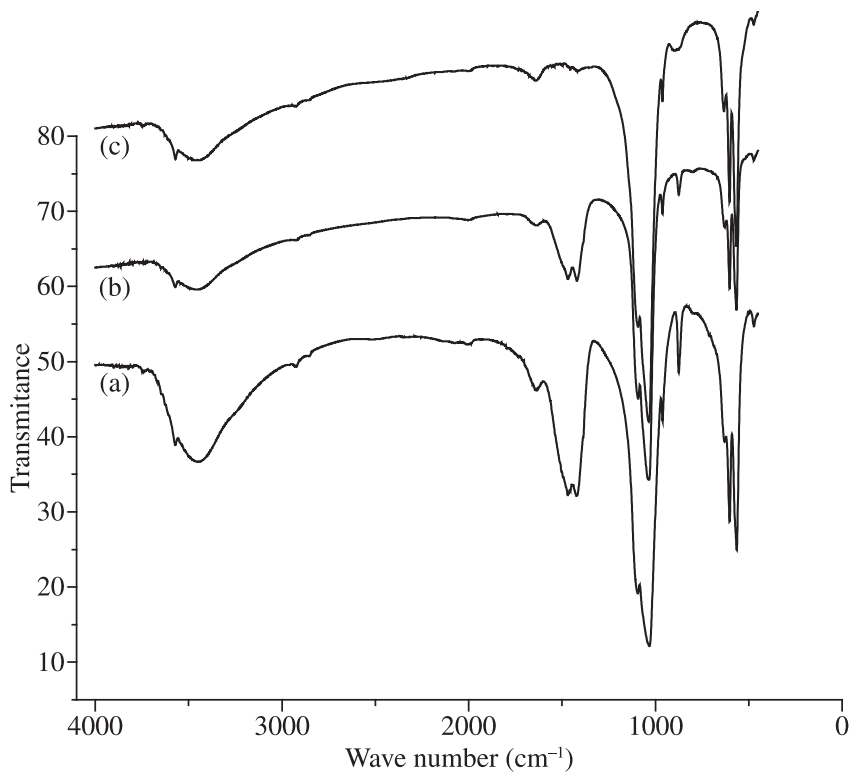

Figure 3. FTIR spectra for HAP particles synthesized in liquid crystal phases formed from a) Renex $-60^{\mathrm{TM}}$, b) Renex $-100^{\mathrm{TM}}$ and c) for commercial hydroxyapatite.

Table 1. Average diameter and $\mathrm{Ca} / \mathrm{P}$ ratio for HAP rod-like particles.

\begin{tabular}{ccc}
\hline Phase & Average diameter of rod-like particles/nm & Ca/P ratio \\
\hline LCR60 & 50 & 2.2 \\
LCR100 & 100 & 2.2 \\
\hline
\end{tabular}

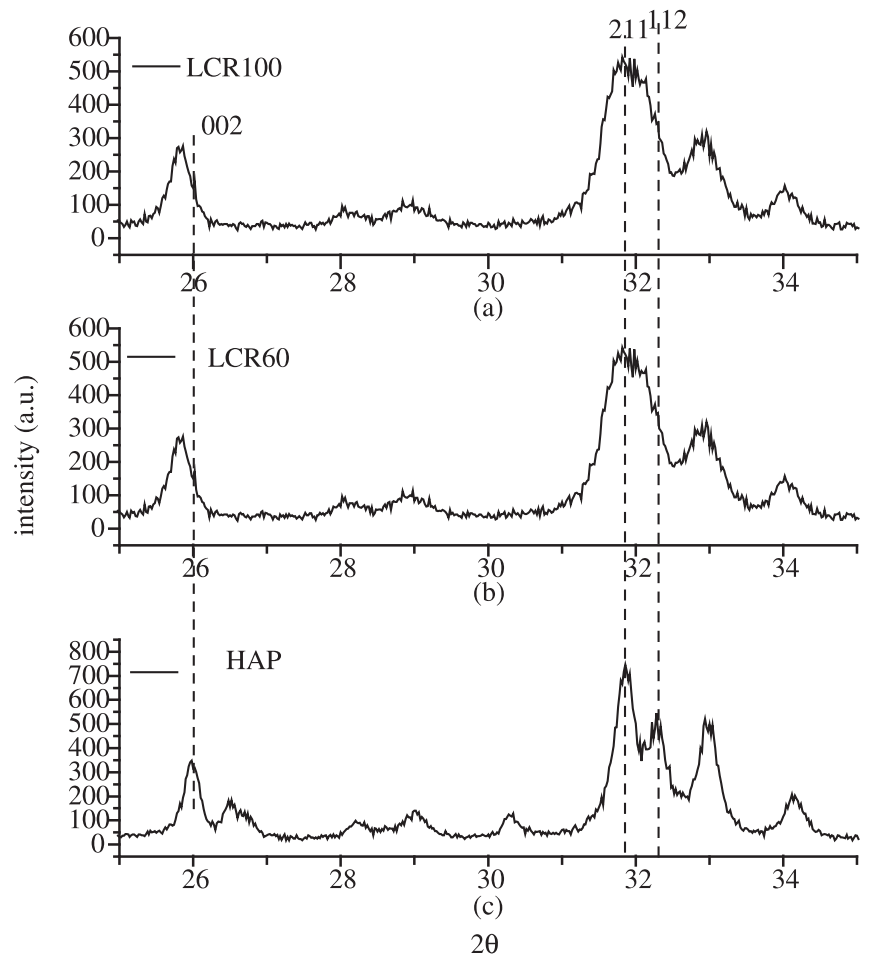

Characterization of phases was also carried out by ${ }^{2} \mathrm{H}-\mathrm{NMR}$ before they were permeated with ammonia and thus before HAP particles formed. The ${ }^{2} \mathrm{H}$ quadrupolar splitting detected in phases LCR60 and LCR100 (Figure 2), indicates that these phases are anisotropic ${ }^{8}$. The results obtained allow confirming that both phases as liquid crystals.

\subsection{Characterization of calcium phosphates}

The composition of the calcium phosphate particles was determined by ICP-OES and FTIR. The phases present in the particles were determined by XRD, also used for an assessment of the crystallinity of the material.

FTIR spectra of both samples (Figure 3 ) are similar to that of commercial HAP (Fluka). Bands at 563, 603, 635, 1035 and $1097 \mathrm{~cm}^{-1}$ were attributed to the vibrational modes $v_{3}$ and $v_{4}$ of phosphate and are characteristic of this compound. Besides the bands typical of phosphate, samples also presented bands at 1420, 1468 and $1642 \mathrm{~cm}^{-1}$, attributed to the vibrational mode $v_{3}$ of carbonate ${ }^{9}$, which allows characterizing the samples as carbonated HAP.

X-Ray diffractograms of both materials (Figure 4) were compared to that of commercial HAP and also to a diffractogram from the databank Powder Diffraction File, International Centre for Diffraction Data, 1997 (card data 9-0432). No other phase was detected besides HAP. The shifting of reflections 002 and 004 towards smaller angles, as well as the overlapping of reflexions 211 and 212, detected in both samples, indicate carbonation of $\mathrm{HAP}^{10}$.

The $\mathrm{Ca} / \mathrm{P}$ ratio determined by ICP-OES was 2.2 for both materials (Table 1). A Ca/P ratio superior to 1.67 , i.e. above the ratio which corresponds to HAP, supports the hypothesis that some phosphate ions are substituted by carbonate ions. This is in good agreement with the results obtained by FTIR and XRD, which indicate that the HAP

Figure 4. X-ray diffractograms for the rod-like particles synthesized in liquid crystal phases formed from a) Renex-100 ${ }^{\mathrm{TM}}$, b) Renex-60 $0^{\mathrm{TM}}$ and c) for commercial hydroxyapatite.

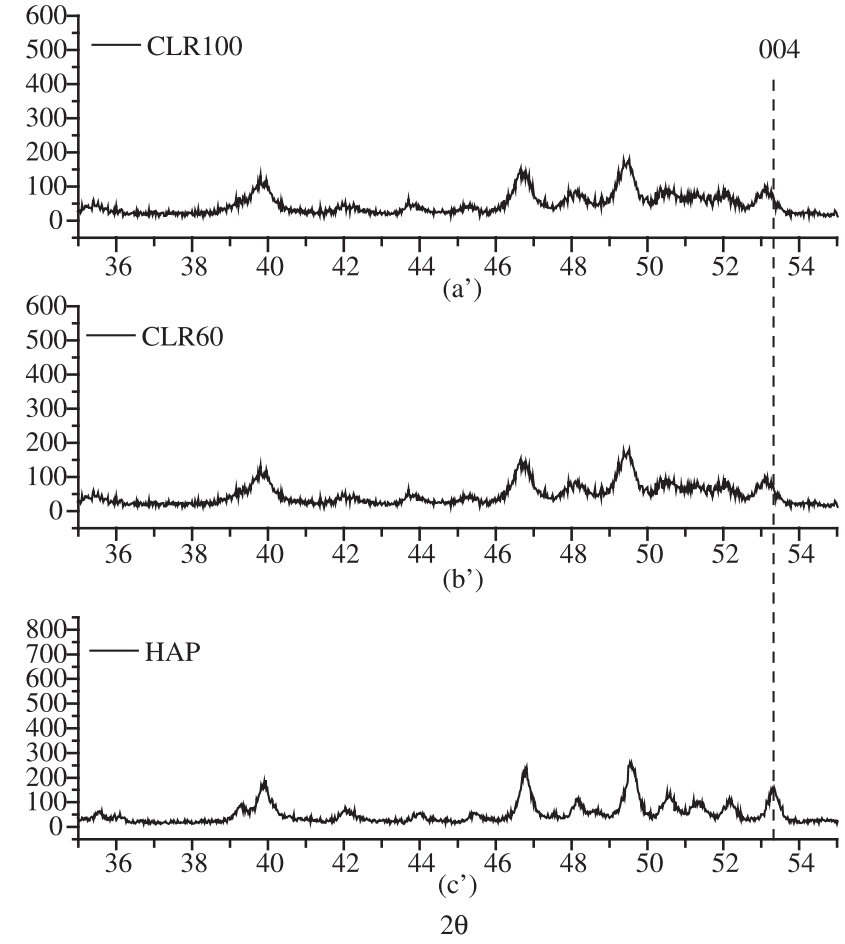




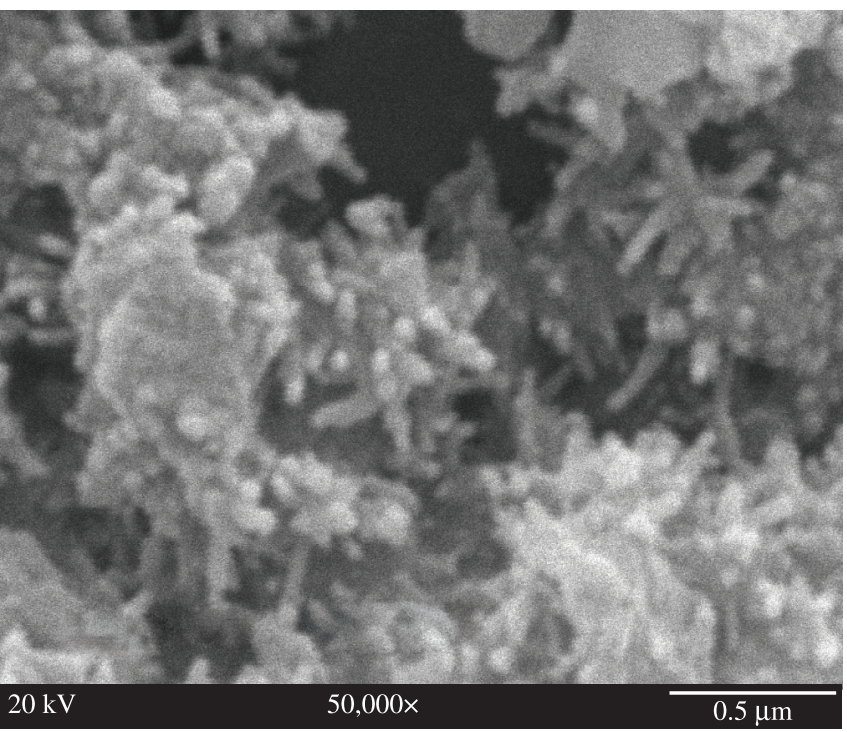

(a)



(b)

Figure 5. SEM micrographs of particles synthesized in the liquid crystal phases with: a) Renex- $60^{\mathrm{TM}}$ and b) Renex-100 ${ }^{\mathrm{TM}}$.

obtained is carbonated. Even though the original mixture used in the preparation of liquid-crystalline phases contained no carbonate ions, these may have been formed and incorporated during the thermal processes that the liquid crystalline phases went through.

The micrographs obtained by SEM of the HAP particles (Figure 5) show that these particles are rod-shaped and approximately $200 \mathrm{~nm}$ in length. Micrographs also show that these nanorods are formed from a linear agglomeration of rounded HAP particles. Table 1 shows the mean diameters of the nanorods produced in both phases.

Figure 5 and Table 1 show that synthesized nanorods in phase LCR60 are narrower than those obtained in phase LCR100. Regardless of the phase used in the synthesis of HAP, the micrographs show that nanorods are formed by particles agglomerated along a line. Since the difference between the two phases lies only in the type of surfactant utilized, more precisely, on the size of the polar chain of the surfactant, these results suggest that liquid crystals act as templates in the synthesis of HAP, in a way that the longer the ethoxylate chain of the surfactant, the larger will be the mean diameter of particles. This may indicate that for the synthesis carried out in liquid crystals, larger hydrophilic surfactant chains may result in larger hydrophilic nanoreactors, which will also form larger particles. These results also show that liquid crystals are an efficient medium for the synthesis of HAP particles similar to those found in bone.

\section{Conclusions}

Liquid crystals formed from Renex ${ }^{\mathrm{TM}}$, cyclohexane and a salts solution were used in order to produce HAP nanorods of about $200 \mathrm{~nm}$ in length and both 50 and $100 \mathrm{~nm}$ in diameter, from phases containing Renex- $60^{\mathrm{TM}}$ and Renex- $100^{\mathrm{TM}}$, respectively. This work shows that the length of the polar chain of the surfactant has a direct effect upon the diameter of particles obtained. The morphological and dimensional homogeneity of particles shows that liquid crystals separate the inorganic phase and control its properties.

\section{Acknowledgements}

Support from the National Council for Scientific and Technological Development (CNPq) is gratefully acknowledged.

\section{References}

1. Jacob DE, Soldati AL, Wirth R, Huth J, Wehrmeister U and Hofmeister W. Nanostructure, composition and mechanisms of bivalve shell growth. Geochimica et Cosmochimica Acta 2008; 72(22):5401-5415.

2. Murugan R and Ramakrishna S. Development of nanocomposites for bone grafting. Composites Science and Technology 2005; 65(15-16):2385-2406.

3. Gajjeraman S, Narayanan K, Hao J, Qin C and George A. Matrix macromolecules in hard tissues control the nucleation and hierarchical assembly of hydroxyapatite. Journal of Biological Chemistry 2007; (2):1193-1204.

4. Allegretti LJM and Bertran CA. The effect of collagen matrix on calcium phosphate mineralization. Key Engineering Materials 2009; (396-398):195-198.

5. Bradt JH, Mertig M, Teresiak A and Pompe W. Biomimetic mineralization of collagen by combined fibril assembly and calcium phosphate formation. Chemistry of Materials 1999; (11):2694-2701.

6. Osawa H, Hoshi $\mathrm{K}$ and Amizuka N. Current concepts of bone biomineralization. Journal of Oral Biosciences 2008; 50(1):1-14.

7. Bertazzo S, Bertran CA and Kawashi EY. Synthetic calcium phosphate nanoparticles mimetic of bone mineral: similarities in composition and morphology. Key Engineering Materials 2006; 309-311(May 2006):507-510.

8. Marsden SS and McBain JW. Aqueous system of non-ionic detergents as studied by x-ray diffraction. The Journal of Physical and Colloid Chemistry 1948; 52(1):110-130.

9. Rehman I and Bonfield W. Characterization of hydroxyapatite and carbonated apatite by photo acoustic FTIR spectroscopy. Journal of Materials Science-Materials in Medicine 1997; 8(1):1-4.

10. LeGeros RZ and Trautz OR. Apatite Crystallites: Effects of carbonate on morphology. Science 1967; 155(3768):1409-1411. 